\title{
3-Loop Corrections to the Heavy Flavor Wilson Coefficients in Deep-Inelastic Scattering*
}

J. Ablinger ${ }^{1}$, A. Behring ${ }^{2}$, J. Blümlein ${ }^{22}$, A. De Freitas ${ }^{2}$, A. Hasselhuhn ${ }^{3}$, A. von Manteuffel ${ }^{4}$, C.G. Raab ${ }^{1,5}$, M. Round ${ }^{1,2}$, C. Schneider ${ }^{1}$, and F. Wißbrock ${ }^{1,2,6}$

${ }^{1}$ Research Institute for Symbolic Computation (RISC), Johannes Kepler University, Altenbergerstraße 69, A-4040, Linz, Austria

${ }^{2}$ Deutsches Elektronen-Synchrotron, DESY, Platanenallee 6, D-15738 Zeuthen, Germany E-mail: johannes.bluemlein@desy.de

${ }^{3}$ Institut für Theoretische Teilchenphysik, Karlsruher Institut für Technologie (KIT), D-76128 Karlsruhe, Germany

${ }^{4}$ PRISMA Cluster of Excellence, Institute of Physics, J. Gutenberg University, D-55099 Mainz, Germany.

${ }^{5}$ Johann Radon Institut (RICAM), Altenbergerstraße 69, A-4040 Linz, Austria

${ }^{6}$ IHES, 35 Route de Chartres, F-91440 Bures-sur-Yvette, France

A survey is given on the status of 3-loop heavy flavor corrections to deep-inelastic structure functions at large enough virtualities $Q^{2}$.

The European Physical Society Conference on High Energy Physics 22-29 July 2015

Vienna, Austria

*This work was supported in part by the Austrian Science Fund (FWF) grants P 27229, SFB F50 (F5009-N15), FP7 ERC Starting Grant 257638 PAGAP and the European Commission through contract PITN-GA-2012-316704 (HIGGSTOOLS).

†Speaker. 


\section{Introduction}

The determination of fundamental parameters of the Standard Model such as the strong coupling constant $\alpha_{s}\left(M_{Z}^{2}\right)$ [1] and the mass of the charm quark $m_{c}$ [2] from the precision World data on unpolarized deep-inelastic lepton-nucleon scattering requires the complete 3-loop QCD corrections. While the massless 3-loop corrections are known [3-5], the calculation of the massive corrections at higher values of $Q^{2} / m_{c}^{2} \gtrsim 10$ is underway [6]. Here $q$ denotes the 4-momentum transfer to the hadronic system and $Q^{2}=-q^{2}$. The 2-loop corrections are known in semi-numerical form [7] ${ }^{1}$ and for large values of $Q^{2}, Q^{2} / m_{c}^{2} \gtrsim 10$ also in analytic form [9,10]. A series of Mellin moments of the 3-loop massive corrections has been computed in Ref. [11] for the operator matrix elements (OMEs) and Wilson coefficients contributing to the structure function $F_{2}\left(x, Q^{2}\right)$ mapping the problem to massive tadpole-structures, which have been calculated using MATAD [12]. All logarithmic terms to 3-loop order have been calculated both for the massive Wilson coefficients as well as for the 3-loop transition matrix elements in the variable flavor number scheme (VFNS) in Ref. [13], referring also to the massive 2-loop OMEs up to $O(\varepsilon)[14,15]$, the 3-loop anomalous dimensions [3,4], and the 2-loop massless Wilson coefficients [16]. At 3-loop order all $T_{F}^{2} N_{F}$-terms have been calculated [17], as well as the $T_{F}^{2}$-terms for the gluonic OMEs [18]. The asymptotic 3-loop contributions to the structure function $F_{L}\left(x, Q^{2}\right)$ were calculated in $[13,19]$ and charged current corrections up to 2-loop order were given in [20]. In a series of technical papers we presented details of the calculation technologies used [21-23] and the properties of mathematical structures occurring [24-36].

In this note a survey is given on the status of the 3-loop heavy flavor corrections to deepinelastic structure functions, with emphasis on recent developments. There are five different contributions to the structure functions $F_{2, L}\left(x, Q^{2}\right)$ from 3-loop order onward. So far four of them have been completed and all the iterative-integral (over general alphabets) contributions to the Wilson coefficient $H_{2, g}^{(3)}$ in the contributing master integrals have been calculated. In the VFNS, eight different OMEs are relevant (counting also transversity), out of which seven have been completed. Here most recently we obtained a representation of the Mellin moments for general even integer values of $N$ of the OME $A_{g g}^{(3)}$ [37].

After a brief introduction into the formalism, we discuss a series of aspects of the calculation of master integrals and present then numerical results on the different heavy flavor Wilson coefficients and OMEs contributing to the structure function $F_{2}\left(x, Q^{2}\right)$, [38-42]. While the former results presented refer to the case of $N_{F}$ massless and a single heavy quark, we also briefly comment on some analytic results for two massive flavors.

\section{Basic Formalism}

In the leading-twist approximation the deep-inelastic structure functions $F_{2, L}\left(x, Q^{2}\right)$ factorize into the non-perturbative parton distribution functions $f_{j}$ and the Wilson coefficients $\mathbb{C}_{j,(2, L)}$, which can

\footnotetext{
${ }^{1}$ For an implementation in Mellin space, see [8].
} 
be calculated perturbatively [43],

$$
F_{(2, L)}\left(x, Q^{2}\right)=\sum_{j} \underbrace{\mathbb{C}_{j,(2, L)}\left(x, \frac{Q^{2}}{\mu^{2}}, \frac{m^{2}}{\mu^{2}}\right)}_{\text {perturbative }} \otimes \underbrace{f_{j}\left(x, \mu^{2}\right)}_{\text {nonpert. }} .
$$

Here $x$ denotes the Bjorken variable $x=Q^{2} / S y$, with $S$ the cms energy squared and $y=2 P . q / S$ the inelasticity, $P$ the proton 4-momentum, $\mu$ denoting both the renormalization and factorization scale, and $\otimes$ denotes the Mellin convolution

$$
f(x) \otimes g(x) \equiv \int_{0}^{1} d y \int_{0}^{1} d z \delta(x-y z) f(y) g(z) .
$$

The Wilson coefficients contain massless $\left(C_{j,(2, L)}\right)$ and massive $\left(H_{j,(2, L)}\right)$ contributions, written here in Mellin space

$$
\mathbb{C}_{j,(2, L)}\left(N, \frac{Q^{2}}{\mu^{2}}, \frac{m^{2}}{\mu^{2}}\right)=C_{j,(2, L)}\left(N, \frac{Q^{2}}{\mu^{2}}\right)+H_{j,(2, L)}\left(N, \frac{Q^{2}}{\mu^{2}}, \frac{m^{2}}{\mu^{2}}\right) .
$$

At large scales $Q^{2}$ the massive contributions

$$
H_{j,(2, L)}\left(N, \frac{Q^{2}}{\mu^{2}}, \frac{m^{2}}{\mu^{2}}\right)=\sum_{i} C_{i,(2, L)}\left(N, \frac{Q^{2}}{\mu^{2}}\right) A_{i j}\left(\frac{m^{2}}{\mu^{2}}, N\right)
$$

factorize [9] into the massless Wilson coefficients and the massive OMEs $A_{i j}$. We are performing the analytic calculation of these quantities and obtain the massive Wilson coefficients at large enough scales $Q^{2}$ to $F_{2}\left(x, Q^{2}\right)$, for $Q^{2} / m^{2} \approx 10$ at the $1 \%$ level. The explicit representation of the Wilson coefficients and the transition formulae in the VFNS for $N_{F} \rightarrow N_{F}+1$ massless quarks to 3-loop order has been given in Ref. [11]. The OMEs having been calculated so far allow to represent

$$
\begin{aligned}
f_{k}\left(N_{F}+1, \mu^{2}\right)+f_{\bar{k}}\left(N_{F}+1, \mu^{2}\right)= & A_{q q, Q}^{\mathrm{NS}}\left(N_{F}, \frac{\mu^{2}}{m^{2}}\right) \otimes\left[f_{k}\left(N_{F}, \mu^{2}\right)+f_{\bar{k}}\left(N_{F}, \mu^{2}\right)\right] \\
& +\tilde{A}_{q q, Q}^{\mathrm{PS}}\left(N_{F}, \frac{\mu^{2}}{m^{2}}\right) \otimes \Sigma\left(N_{F}, \mu^{2}\right)+\tilde{A}_{q g, Q}^{\mathrm{S}}\left(N_{F}, \frac{\mu^{2}}{m^{2}}\right) \otimes G\left(N_{F}, \mu^{2}\right) \\
G\left(N_{F}+1, \mu^{2}\right)= & A_{g q, Q}^{\mathrm{S}}\left(N_{F}, \frac{\mu^{2}}{m^{2}}\right) \otimes \Sigma\left(N_{F}, \mu^{2}\right)+A_{g g, Q}^{S}\left(N_{F}, \frac{\mu^{2}}{m^{2}}\right) \otimes G\left(N_{F}, \mu^{2}\right)
\end{aligned}
$$

the transition in the non-singlet case and for the gluon distribution to 3-loop order. Here, $f_{k}$ denotes the $k$ th massless quark densities, $\Sigma=\sum_{k}\left(f_{k}+\bar{f}_{k}\right)$ the singlet- and $G$ the gluon distribution function.

We turn now to the analytic calculation of the OMEs.

\section{Calculation of the Master Integrals}

Due to the large number of integrals being associated to the Feynman integrals contributing to the eight principal OMEs, we use the integration-by-parts relations (IBPs) [44] implemented in the package Reduze2 $[45,46]$ and reduce them to master integrals. An overview on the respective numbers is given in the following Table. 


\begin{tabular}{|l|r|r|r|}
\hline & Diagrams & Integrals & Masters \\
\hline$A_{q q, Q}^{\mathrm{NS}[\mathrm{TR}](3)}$ & 110 & 5636 & 35 \\
$A_{g q, Q}^{(3)}$ & 86 & 12253 & 41 \\
$A_{Q q}^{\mathrm{PS}(3)}$ & 125 & 4824 & 66 \\
$A_{g g, Q}^{(3)}$ & 642 & 68131 & 139 \\
$A_{Q g}^{(3)}$ & 1233 & 34123 & 340 \\
\hline
\end{tabular}

For the calculation of the master integrals different analytic technologies are used, see Refs. [23, 47]. The simplest topologies can be represented by generalized hypergeometric functions and their extensions [48] and expanded in the dimensional parameter $\varepsilon$ leading to nested finite and infinite sums. In more involved cases, Mellin-Barnes representations [49] need to be used in addition, which also lead to multiple sum representations. In case of Feynman diagrams without singularities in $\varepsilon$ also the method of hyperlogarithms [50-52] can be directly applied. The next general class of cases can be solved using the method of differential equations [53] for linear systems of master integrals. In the present calculation one auxiliary parameter emerges due to the formal resummation of the local operator insertion into propagator terms, the simplest example being

$$
\sum_{n=0}^{\infty} x^{n}(\Delta \cdot p)^{n} \rightarrow \frac{1}{1-x \Delta \cdot p} .
$$

In Refs. [23,47] we have developed an algorithm for solving these systems in the form they appear in the calculation with no need to choose a special basis [54]. The differential system is cast into a higher order difference equation by using a decoupling algorithm [55] and then solved using the methods encoded in the package Sigma [56,57]. The remaining equations of the system are solved subsequently as linear equations. The corresponding initial values are obtained for fixed Mellin moments and are obtained from the equations given by the IBPs. In some cases it is advantageous to compute the given multiple integrals using the (multivariate) Almkvist-Zeilberger Theorem [58] as integration method.

Most of the above methods lead to nested finite and infinite sums after expanding in the dimensional parameter $\varepsilon$, which again have to be solved using modern summation technologies [59-67], encoded in the packages Sigma [56,57], EvaluateMultiSums, SumProduction [68], SolveCoupledSystem [23], $\rho$ SUM [69], and Multi Integrate [70]. Moreover, mutual use is made of the package HarmonicSums [33-35,70,71]. The differential equation method leads to sequential difference equations in a different way, which are also solved using difference-field theory.

Using these methods seven out of eight OMEs could be completely calculated. In fact we could calculate all master integrals also contributing to $A_{Q g}^{(3)}$, that lead to iterative integrals over whatever alphabet. There remain 105 master integrals containing non-iterative integral parts, forming classes of a new kind.

\section{Numerical Results}

In the following we would like to present some numerical results for the massive Wilson coefficients contributing to the structure function $F_{2}\left(x, Q^{2}\right)$ at larger values of $Q^{2}$ up to 3-loop order in the strong coupling constant $a_{s}=\alpha_{s} /(4 \pi)$ and for OMEs in the VFNS. 

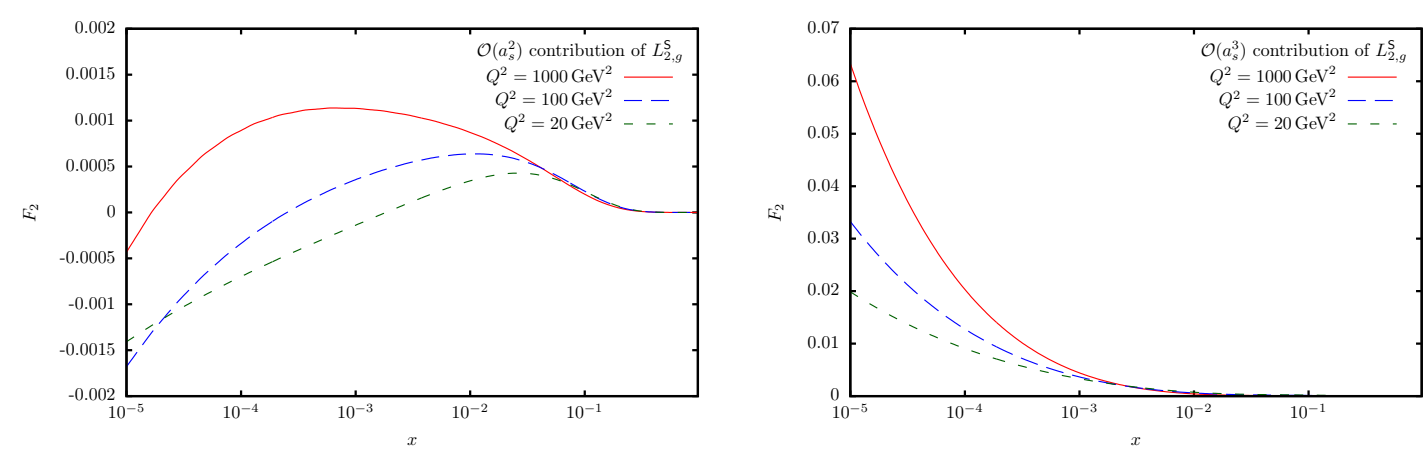

Figure 1: Left panel: the contribution of the Wilson coefficient $L_{2, g}^{S}$ at $O\left(a_{s}^{2}\right)$. Right panel: the $O\left(a_{s}^{3}\right)$ contributions; from Ref. [13].

In Figure 1 we we illustrate the contributions up to $O\left(a_{s}^{2}\right)$ and $O\left(a_{s}^{3}\right)$ to the structure function $F_{2}\left(x, Q^{2}\right)$ referring to the parton distribution functions [72] here and in the following and $m_{c}=$ $1.59 \mathrm{GeV}$ in the on-shell scheme [2]. It turns out that the 2-loop corrections are even smaller than those at 3-loops because of a small $x$ effect contributing from 3-loops onward.
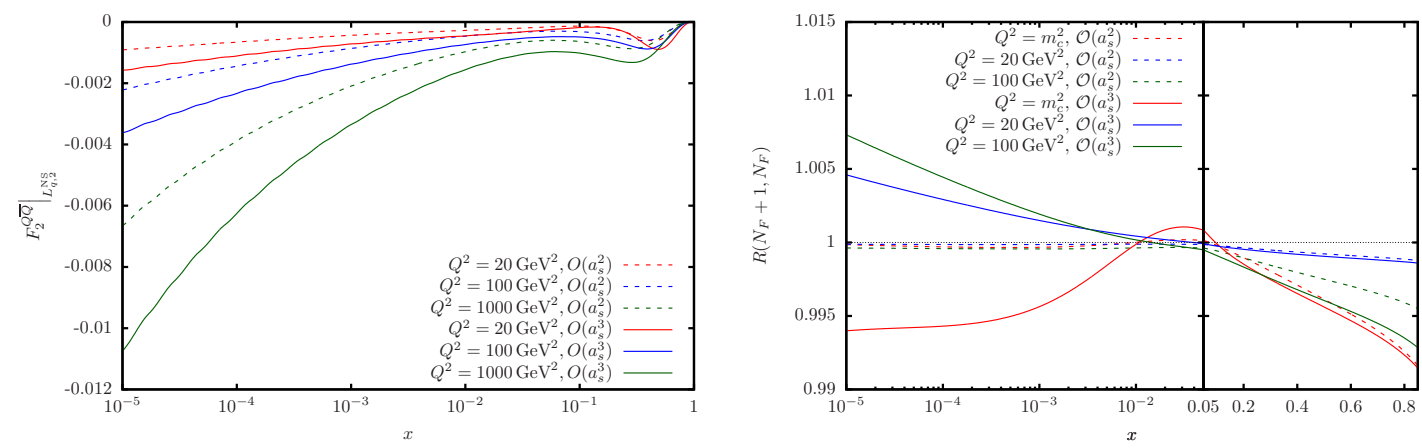

Figure 2: Left panel: The massive flavor non-singlet contributions to $F_{2}\left(x, Q^{2}\right)$. Right panel: The ratio of the non-singlet $u+\bar{u}$-quark contribution of 4- to 3 flavors as a function of $x$ for different matching scales; from Ref. [41].

In Figure 2 we illustrate the 2- and 3-loop flavor non-singlet heavy flavor contributions to the structure function $F_{2}\left(x, Q^{2}\right)$ as a function of $x$ and $Q^{2}$. The 3-loop corrections lower the contributions obtained at 2-loops. The corrections are of the $1 \%$ level. In the flavor non-singlet case all transition functions in the VFNS are available, and we illustrate also the ratio of the non-singlet $u+\bar{u}$-quark contribution of 4- to 3 flavors as a function of $x$ for different matching scales. While at small $x$ the 2-loop effects are tiny, the 3-loop effects show some dependence both at small and larger values of $x$.

Turning to the polarized case, we illustrate in Figure 3 the ratio of the charm contribution to the massless contributions to $g_{1}\left(x, Q^{2}\right)$ as a function of $x$ and $Q^{2}$ up to 3-loop order. It turns out to be positive in the region of lower $x$. The correction becomes negative at large values of $x$ and amounts to a few percent. Similarly, we have calculated the 3-loop heavy flavor corrections to the non-singlet structure function $x\left(F_{3}^{W^{+}}+F_{3}^{W^{-}}\right)$. Here the heavy flavor correction varies from $+3 \%$ to $-4 \%$ from small to large $x$. 

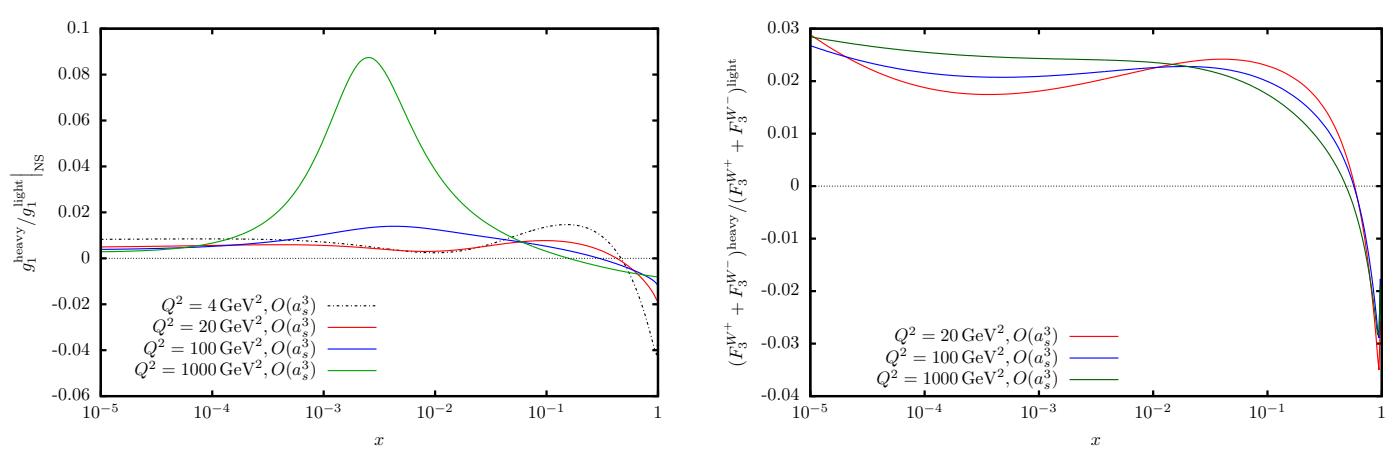

Figure 3: Left panel: The ratio of the charm to the massless flavor contribution to the polarized structure function $g_{1}\left(x, Q^{2}\right)$ up to 3-loop order; from Ref. [39]. Right panel: The ratio of the charm to the massless flavor contribution to the structure function $x\left(F_{3}^{W^{+}}+F_{3}^{W^{-}}\right)$up to 3-loop order; from Ref. [38].
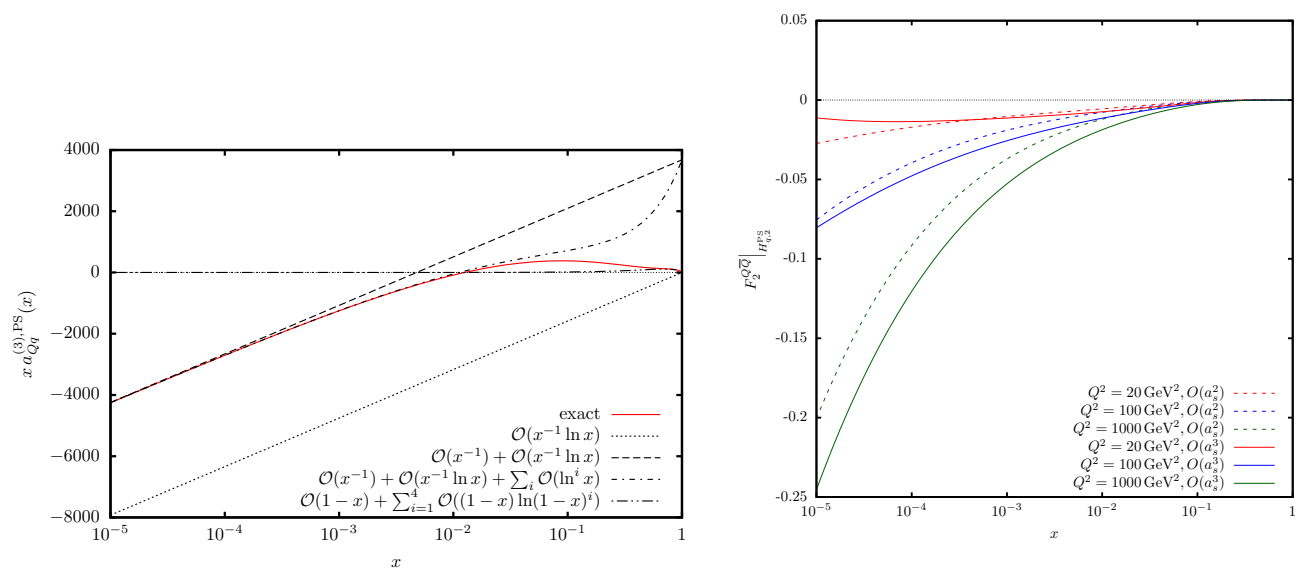

Figure 4: Left panel: $x a_{Q q}^{(3), P S}(x)$ in the low $x$ region (solid red line) and leading terms approximating this quantity; dotted line: 'leading' small $x$ approximation $O(\ln (x) / x)$, dashed line: adding the $O(1 / x)$-term, dash-dotted line: adding all other logarithmic contributions. Right panel: Charm pure singlet contribution to $F_{2}\left(x, Q^{2}\right)$ to $O\left(a_{s}^{2}\right)$ and $O\left(a_{s}^{3}\right)$ as a function of $Q^{2}$; from Ref. [40].

In Figure 4 we illustrate the 3-loop pure-singlet corrections to the structure function $F_{2}\left(x, Q^{2}\right)$. First we consider the constant part of the unrenormalized OME as a function of $x$. There is a prediction of the most singular small $x$ contribution $[73,74]$, which is analytically confirmed in our calculation. However, this term is misleading and describes this correction nowhere, since subleading terms are of the same size and are therefore important, cf. [75]. The massive puresinglet corrections are larger than those in the non-singlet case and again the 3-loop corrections are relevant.

\section{5. $\mathbf{A}_{\mathbf{g g}}(\mathbf{N})$}

Most recently we have calculated the OME $A_{g g}(N)$ at 3-loop order contributing in the VFNS. In a first step we calculated this OME for all its even integer moments for $N \geq 2$. Here the part not induced by renormalization and factorization is the constant part of the 3-loop unrenormalized 
matrix element. Its principal structure is given by

$$
\begin{aligned}
& a_{g g, Q}^{(3)}=\frac{1+(-1)^{N}}{2}\left\{C _ { F } ^ { 2 } T _ { F } \left[\frac{16\left(N^{2}+N+2\right)}{N^{2}(N+1)^{2}} \sum_{i=1}^{N} \frac{\left(\begin{array}{c}
2 i \\
i
\end{array}\right)\left(\begin{array}{c}
i \\
\left.\sum_{j=1}^{i} \frac{4^{j} S_{1}(j-1)}{\left(\begin{array}{c}
(j) \\
j
\end{array}\right) j^{2}}-7 \zeta_{3}\right)
\end{array}\right.}{4^{i}(i+1)^{2}}\right.\right. \\
& -\frac{4 P_{69} S_{1}^{2}}{3(N-1) N^{4}(N+1)^{4}(N+2)}+\tilde{\gamma}_{g q}^{(0)}\left(\frac{128\left(S_{-4}-S_{-3} S_{1}+S_{-3,1}+2 S_{-2,2}\right)}{3 N(N+1)(N+2)}\right. \\
& \left.\left.+\frac{4\left(5 N^{2}+5 N-22\right) S_{1}^{2} S_{2}}{3 N(N+1)(N+2)}+\cdots\right)+\cdots\right] \\
& +C_{A} C_{F} T_{F}\left[\frac{16 P_{42}}{3(N-1) N^{2}(N+1)^{2}(N+2)} \sum_{i=1}^{N} \frac{\left(\begin{array}{c}
2 i \\
i
\end{array}\right)\left(\begin{array}{c}
i \\
\left.\sum_{j=1} \frac{4^{j} S_{1}(j-1)}{\left(\begin{array}{c}
2 j \\
j
\end{array}\right) j^{2}}-7 \zeta_{3}\right)
\end{array}\right.}{4^{i}(i+1)^{2}}\right. \\
& +\frac{32 P_{2} S_{-2,2}}{(N-1) N^{2}(N+1)^{2}(N+2)}-\frac{64 P_{14} S_{-2,1,1}}{3(N-1) N^{2}(N+1)^{2}(N+2)} \\
& \left.-\frac{16 P_{23} S_{-4}}{3(N-1) N^{2}(N+1)^{2}(N+2)}+\frac{4 P_{63} S_{4}}{3(N-2)(N-1) N^{2}(N+1)^{2}(N+2)}+\cdots\right] \\
& +C_{A}^{2} T_{F}\left[-\frac{4 P_{46}}{3(N-1) N^{2}(N+1)^{2}(N+2)} \sum_{i=1}^{N} \frac{\left(\begin{array}{c}
2 i \\
i
\end{array}\right)\left(\begin{array}{c}
i \\
\left.\sum_{j=1}^{i} \frac{4^{j} S_{1}(j-1)}{\left(\begin{array}{c}
2 j \\
j
\end{array}\right) j^{2}}-7 \zeta_{3}\right)
\end{array}\right.}{4^{i}(i+1)^{2}}\right. \\
& +\frac{256 P_{5} S_{-2,2}}{9(N-1) N^{2}(N+1)^{2}(N+2)}+\frac{32 P_{30} S_{-2,1,1}+16 P_{35} S_{-3,1}+16 P_{44} S_{-4}}{9(N-1) N^{2}(N+1)^{2}(N+2)} \\
& \left.+\frac{16 P_{52} S_{-2}^{2}}{27(N-1) N^{2}(N+1)^{2}(N+2)}+\frac{8 P_{36} S_{2}^{2}}{9(N-1) N^{2}(N+1)^{2}}+\cdots\right] \\
& +C_{F} T_{F}^{2}\left[-\frac{16 P_{48}\left(\begin{array}{c}
2 N \\
N
\end{array}\right) 4^{-N}\left(\sum_{i=1}^{N} \frac{4^{i} S_{1}(i-1)}{\left(\begin{array}{c}
2 i \\
i
\end{array} i^{2}\right.}-7 \zeta_{3}\right)}{3(N-1) N(N+1)^{2}(N+2)(2 N-3)(2 N-1)}\right. \\
& -\frac{32 P_{86} S_{1}}{81(N-1) N^{4}(N+1)^{4}(N+2)(2 N-3)(2 N-1)} \\
& \left.\left.+\frac{16 P_{45}\left(S_{1}^{2}-S_{2} / 3\right)}{27(N-1) N^{3}(N+1)^{3}(N+2)}+\cdots\right]+\cdots\right\} \text {, }
\end{aligned}
$$

displaying some of the new nested sums, with $P_{j}$ polynomials in $N$. The contributions $\propto N_{F} T_{F}^{2}$ or $T_{F}^{2}$ were calculated before. Also, with this calculation we have rederived all the contributions to the 3-loop anomalous dimensions $\propto T_{F}$.

\section{Analytic Two-Mass Contributions}

In the previous sections we have discussed the case of $N_{F}$ massless and one massive quark. Starting with 3-loop order, there are also graphs that contain two massive lines of different mass. The usual 
VFNS, cf. e.g. [11], is therefore no longer applicable and one cannot define individual heavy flavor parton densities anymore. This is due to the fact that the ratio $\eta=m_{c}^{2} / m_{b}^{2}$ is $\sim 1 / 10$ only and charm cannot be considered to be massless already at the bottom mass scale. In $[51,76]$ we have calculated the moments $N=2,4,6$ for all contributing OMEs to a precision of better than $10^{-4}$ using the package $q 2 e$ [77]. The complete results in the non-singlet case and for all contributing scalar integrals to $A_{g g}^{(3)}$ have been calculated in $[51,78]$. Here various new iterated integrals over root-valued letters appear with also the variable $\eta$ as parameter. Calculating these corrections for general values of the Mellin variable $N$, it is not always possible to expand in the parameter $\eta$. We therefore have calculated the complete corrections analytically in these cases.

\section{Conclusions}

In 2009 a series of Mellin moments up to $N=10-14$ for all massive 3-loop OMEs and Wilson coefficients have been calculated, followed by the computation of the first two massive 3-loop Wilson coefficients for $F_{2}\left(x, Q^{2}\right), L_{q}^{(3), \mathrm{PS}}(N)$ and $L_{g}^{(3), \mathrm{S}}(N)$, in the asymptotic region in 2010, completing soon after all contributions $\propto N_{F} T_{F}^{2}$. The logarithmic contributions are completely known. Ladder, $V$-Graph and Benz-topologies for graphs, with no singularities in $\varepsilon$, were systematically calculated for general values of $N$ in 2013. The corresponding case of singular structures and associated physical diagrams has been solved in 2015. Here new functions occur, including iterated integrals with a larger number of root-valued letters of quadratic forms. In 2014 we calculated, based on the technologies described in Section 3, the 3-loop Wilson coefficients and massive OMEs $L_{q}^{\mathrm{NS},(3)}$, $A_{g q, Q}^{\mathrm{S},(3)}, A_{q q, Q}^{\mathrm{NS}, \mathrm{TR}(3)}, H_{2, q}^{\mathrm{PS}(3)}$ and $A_{Q q}^{\mathrm{PS}(3)}$. A method for the calculation of graphs with two massive lines of equal masses and operator insertions has been developed and applied to $A_{g g, Q}^{(3)}(N)$. The method can be generalized to the case of unequal masses. Here the moments for $N=2,4,6$ for all graphs with two quark lines of unequal masses are now known, which requires extensions in the renormalization procedure. It turns out in general, that in the case of general values of $N$ one has to compute the complete result, as the expansion in $m_{c}^{2} / m_{b}^{2}$ not always commutes with the functional structure in $N$, unlike the case of fixing $N$ a priori. We also calculated the charged current Wilson coefficients up to $O\left(\alpha_{s}^{2}\right)$ correcting some errors in the foregoing literature.

In all cases the corresponding 3-loop anomalous dimensions were computed as functions of $N$ and $x$ resp., those for transversity for the first time ab initio. The corresponding contributions are $\propto N_{F}^{k}, k \geq 1$, the latest example being $\hat{\gamma}_{g g}^{(3)}$. In the pure singlet case this is the first complete recalculation using a different method than in Ref. [4]. All master integrals for $A_{g g, Q}^{(3)}$ have been computed and the complete expression for general values of $N=2 k, k \in \mathbb{N}, k \geq 1$ has been calculated in 2015. The computation of the $\operatorname{OME} A_{Q g}^{(3)}(N)$ made also some progress. Here all master integrals that can be thoroughly represented as nested sums in difference fields have been calculated. A series of new computer-algebra and mathematical technologies were developed and encoded in the still growing packages Sigma, EvaluateMultiSums, SumProduction, SolveCoupledSystem, $\rho$ Sum, HarmonicSums and Multi Integrate for efficient use. In the present project still more involved structures await their analytic solution. As for those structures having been found in the past we expect that they also appear in other massive higher-loop precision calculations in collider physics. 
Acknowledgment. We would like to thank M. Steinhauser for the possibility to use the package MATAD 3.0.

\section{References}

[1] S. Bethke et al., Workshop on Precision Measurements of $\alpha_{s}$, arXiv:1110.0016 [hep-ph]; S. Moch, S. Weinzierl et al., High precision fundamental constants at the TeV scale, arXiv:1405.4781 [hep-ph];

D. d'Enterria et al., arXiv:1512.05194 [hep-ph].

[2] S. Alekhin, J. Blümlein, K. Daum, K. Lipka and S. Moch, Phys. Lett. B 720 (2013) 172 [arXiv:1212.2355 [hep-ph]].

[3] S. Moch, J.A.M. Vermaseren and A. Vogt, Nucl. Phys. B 688 (2004) 101 [hep-ph/0403192];

[4] A. Vogt, S. Moch and J.A.M. Vermaseren, Nucl. Phys. B 691 (2004) 129 [hep-ph/0404111].

[5] J.A.M. Vermaseren, A. Vogt and S. Moch, Nucl. Phys. B 724 (2005) 3 [hep-ph/0504242].

[6] J. Blümlein, A. De Freitas and C. Schneider, Nucl. Part. Phys. Proc. 261-262 (2015) 185 [arXiv:1411.5669 [hep-ph]].

[7] E. Laenen, S. Riemersma, J. Smith and W.L. van Neerven, Nucl. Phys. B 392 (1993) 162; 229;

S. Riemersma, J. Smith and W. L.van Neerven, Phys. Lett. B 347 (1995) 143 [hep-ph/9411431].

[8] S.I. Alekhin and J. Blümlein, Phys. Lett. B 594 (2004) 299 [hep-ph/0404034].

[9] M. Buza, Y. Matiounine, J. Smith, R. Migneron and W.L. van Neerven, Nucl. Phys. B 472 (1996) 611 [hep-ph/9601302].

[10] I. Bierenbaum, J. Blümlein and S. Klein, Nucl. Phys. B 780 (2007) 40 [hep-ph/0703285].

[11] I. Bierenbaum, J. Blümlein and S. Klein, Nucl. Phys. B 820 (2009) 417 [arXiv:0904.3563 [hep-ph]]; J. Blümlein, S. Klein and B. Tödtli, Phys. Rev. D 80 (2009) 094010 [arXiv:0909.1547 [hep-ph]].

[12] M. Steinhauser, Comput. Phys. Commun. 134 (2001) 335 [hep-ph/0009029].

[13] A. Behring, I. Bierenbaum, J. Blümlein, A. De Freitas, S. Klein and F. Wißbrock, Eur. Phys. J. C 74 (2014) 9, 3033 [arXiv:1403.6356 [hep-ph]].

[14] I. Bierenbaum, J. Blümlein, S. Klein and C. Schneider, Nucl. Phys. B 803 (2008) 1 [arXiv:0803.0273 [hep-ph]].

[15] I. Bierenbaum, J. Blümlein and S. Klein, Phys. Lett. B 672 (2009) 401 [arXiv:0901.0669 [hep-ph]].

[16] W.L. van Neerven and E.B. Zijlstra, Phys. Lett. B 272 (1991) 127; Phys. Lett. B 273 (1991) 476; Nucl. Phys. B 383 (1992) 525;

S. Moch and J.A.M. Vermaseren, Nucl. Phys. B 573 (2000) 853 [hep-ph/9912355].

[17] J. Ablinger, J. Blümlein, S. Klein, C. Schneider and F. Wißbrock, Nucl. Phys. B 844 (2011) 26 [arXiv:1008.3347 [hep-ph]];

J. Blümlein, A. Hasselhuhn, S. Klein and C. Schneider, Nucl. Phys. B 866 (2013) 196 [arXiv:1205.4184 [hep-ph]].

[18] J. Ablinger, J. Blümlein, A. De Freitas, A. Hasselhuhn, A. von Manteuffel, M. Round and C. Schneider, arXiv:1405.4259 [hep-ph], Nucl. Phys. B 885 (2014) 280.

[19] J. Blümlein, A. De Freitas, W.L. van Neerven and S. Klein, Nucl. Phys. B 755 (2006) 272 [hep-ph/0608024]. 
[20] M. Glück, S. Kretzer and E. Reya, Phys. Lett. B 380 (1996) 171 [Erratum: Phys. Lett. B 405 (1997) 391] [hep-ph/9603304];

J. Blümlein, A. Hasselhuhn, P. Kovacikova and S. Moch, Phys. Lett. B 700 (2011) 294 [arXiv:1104.3449 [hep-ph]];

M. Buza and W.L. van Neerven, Nucl. Phys. B 500 (1997) 301 [hep-ph/9702242];

J. Blümlein, A. Hasselhuhn and T. Pfoh, Nucl. Phys. B 881 (2014) 1 [arXiv:1401.4352 [hep-ph]].

[21] J. Ablinger, J. Blümlein, A. Hasselhuhn, S. Klein, C. Schneider and F. Wißbrock, Nucl. Phys. B 864 (2012) 52 [arXiv:1206.2252 [hep-ph]];

[22] J. Ablinger, J. Blümlein, C. Raab, C. Schneider and F. Wißbrock, Nucl. Phys. B 885 (2014) 409 [arXiv:1403.1137 [hep-ph]].

[23] J. Ablinger, A. Behring, J. Blümlein, A. De Freitas, A. von Manteuffel and C. Schneider, arXiv:1509.08324 [hep-ph], Computer Phys. Commun. in print.

[24] J. Blümlein and S. Kurth, Phys. Rev. D 60 (1999) 014018 [arXiv:hep-ph/9810241].

[25] J.A.M. Vermaseren, Int. J. Mod. Phys. A 14 (1999) 2037 [arXiv:hep-ph/9806280].

[26] J. Blümlein, Comput. Phys. Commun. 159 (2004) 19 [hep-ph/0311046].

[27] J. Blümlein, Comput. Phys. Commun. 180 (2009) 2218 [arXiv:0901.3106 [hep-ph]].

[28] J. Blümlein, in : Proceedings of the Workshop Motives, Quantum Field Theory, and Pseudodifferential Operators, Clay Mathematics Institute, Boston University, June 2-13, 2008, Clay Mathematics Proceedings Vol. 12 (2010) 167, eds. A. Carey, D. Ellwood, S. Paycha, S. Rosenberg, [arXiv:0901.0837 [math-ph]].

[29] J. Blümlein, D.J. Broadhurst and J.A.M. Vermaseren, Comput. Phys. Commun. 181 (2010) 582 [arXiv:0907.2557 [math-ph]].

[30] J. Blümlein, M. Kauers, S. Klein and C. Schneider, Comput. Phys. Commun. 180 (2009) 2143 [arXiv:0902.4091 [hep-ph]].

[31] J. Blümlein, S. Klein, C. Schneider and F. Stan, J. Symbolic Comput. 47 (2012) 1267-1289 [arXiv:1011.2656 [cs.SC]].

[32] S. Moch, P. Uwer and S. Weinzierl, J. Math. Phys. 43 (2002) 3363 [hep-ph/0110083].

[33] J. Ablinger, J. Blümlein and C. Schneider, J. Math. Phys. 54 (2013) 082301 [arXiv:1302.0378 [math-ph]].

[34] J. Ablinger, J. Blümlein, C.G. Raab and C. Schneider, J. Math. Phys. 55 (2014) 112301 [arXiv:1407.1822 [hep-th]].

[35] J. Ablinger, J. Blümlein and C. Schneider, J. Math. Phys. 52 (2011) 102301 [arXiv:1105.6063 [math-ph]].

[36] J. Ablinger, J. Blümlein and C. Schneider, J. Phys. Conf. Ser. 523 (2014) 012060 [arXiv:1310.5645 [math-ph]];

J. Ablinger and J. Blümlein, in: Computer Algebra in Quantum Field Theory: Integration, Summation and Special Functions, C. Schneider, J. Blümlein, Eds., p. 1, (Springer, Wien, 2013) [arXiv:1304.7071 [math-ph]].

[37] J. Ablinger et al., DESY 15-112 .

[38] A. Behring, J. Blümlein, A. De Freitas, A. Hasselhuhn, A. von Manteuffel and C. Schneider, Phys. Rev. D 92 (2015) 11, 114005 [arXiv:1508.01449 [hep-ph]]. 
[39] A. Behring, J. Blümlein, A. De Freitas, A. von Manteuffel and C. Schneider, Nucl. Phys. B 897 (2015) 612 [arXiv:1504.08217 [hep-ph]].

[40] J. Ablinger, A. Behring, J. Blümlein, A. De Freitas, A. von Manteuffel and C. Schneider, Nucl. Phys. B 890 (2014) 48 [arXiv:1409.1135 [hep-ph]].

[41] J. Ablinger, A. Behring, J. Blümlein, A. De Freitas, A. Hasselhuhn, A. von Manteuffel, M. Round, C. Schneider, and F. Wißbrock, Nucl. Phys. B 886 (2014) 733 [arXiv:1406.4654 [hep-ph]].

[42] J. Ablinger, J. Blümlein, A. De Freitas, A. Hasselhuhn, A. von Manteuffel, M. Round, C. Schneider and F. Wißbrock, Nucl. Phys. B 882 (2014) 263 [arXiv:1402.0359 [hep-ph]].

[43] J. Blümlein, Prog. Part. Nucl. Phys. 69 (2013) 28 [arXiv:1208.6087 [hep-ph]].

[44] J. Lagrange, Nouvelles recherches sur la nature et la propagation du son, Miscellanea Taurinensis, t. II, 1760-61; Oeuvres t. I, p. 263;

C.F. Gauß, Theoria attractionis corporum sphaeroidicorum ellipticorum homogeneorum methodo novo tractate, Commentationes societas scientiarum Gottingensis recentiores, Vol III, 1813, Werke Bd. V pp. 5-7;

G. Green, Essay on the Mathematical Theory of Electricity and Magnetism, Nottingham, 1828 [Green Papers, pp. 1-115];

M. Ostrogradski, Mem. Ac. Sci. St. Peters., 6, (1831) 39;

K.G. Chetyrkin, A.L. Kataev and F.V. Tkachov, Nucl. Phys. B 174 (1980) 345;

S. Laporta, Int. J. Mod. Phys. A 15 (2000) 5087 [hep-ph/0102033].

[45] C. Studerus, Comput. Phys. Commun. 181 (2010) 1293 [arXiv:0912.2546 [physics.comp-ph]].

[46] A. von Manteuffel and C. Studerus, arXiv:1201.4330 [hep-ph].

[47] J. Ablinger, J. Blümlein, A. De Freitas and C. Schneider, arXiv:1601.01856 [cs.SC].

[48] W.N. Bailey, Generalized Hypergeometric Series, (Cambridge University Press, Cambridge, 1935);

L.J. Slater, Generalized Hypergeometric Functions, (Cambridge University Press, Cambridge, 1966);

P. Appell and J. Kampé de Fériet, Fonctions Hypergéométriques et Hyperspériques, Polynomes

D' Hermite, (Gauthier-Villars, Paris, 1926);

P. Appell, Les Fonctions Hypergëométriques de Plusieur Variables, (Gauthier-Villars, Paris, 1925);

J. Kampé de Fériet, La fonction hypergëométrique,(Gauthier-Villars, Paris, 1937);

H. Exton, Multiple Hypergeometric Functions and Applications, (Ellis Horwood, Chichester, 1976);

H. Exton, Handbook of Hypergeometric Integrals, (Ellis Horwood, Chichester, 1978);

H.M. Srivastava and P.W. Karlsson, Multiple Gaussian Hypergeometric Series, (Ellis Horwood, Chicester, 1985);

M.J. Schlosser, in: Computer Algebra in Quantum Field Theory: Integration, Summation and Special Functions, C. Schneider, J. Blümlein, Eds., p. 305, (Springer, Wien, 2013)

[arXiv:1305.1966 [math.CA]].

[49] E.W. Barnes, Proc. Lond. Math. Soc. (2) 6 (1908) 141; Quart. Journ. Math. 41 (1910) 136;

H. Mellin, Math. Ann. 68 (1910) 305;

M. Czakon, Comput. Phys. Commun. 175 (2006) 559 [hep-ph/0511200];

A.V. Smirnov and V.A. Smirnov, Eur. Phys. J. C 62 (2009) 445 [arXiv:0901.0386 [hep-ph]].

[50] F.C.S. Brown, Commun. Math. Phys. 287 (2009) 925 [arXiv:0804.1660 [math.AG]]. 
[51] F. Wißbrock, $O\left(\alpha_{s}^{3}\right)$ Contributions to the Heavy Flavor Wilson Coefficients of the Structure Function $F_{2}\left(x, Q^{2}\right)$ at $Q^{2} \gg m^{2}, \mathrm{PhD}$ Thesis, TU Dortmund, 2015.

[52] E. Panzer, JHEP 1403 (2014) 071 [arXiv:1401.4361 [hep-th]].

[53] A.V. Kotikov, Phys. Lett. B 254 (1991) 158;

E. Remiddi, Nuovo Cim. A 110 (1997) 1435 [hep-th/9711188];

M. Caffo, H. Czyz, S. Laporta and E. Remiddi, Acta Phys. Polon. B 29 (1998) 2627

[hep-th/9807119]; Nuovo Cim. A 111 (1998) 365 [hep-th/9805118];

T. Gehrmann and E. Remiddi, Nucl. Phys. B 580 (2000) 485 [hep-ph/9912329].

[54] J.M. Henn, Phys. Rev. Lett. 110 (2013) 251601 [arXiv:1304.1806 [hep-th]].

[55] B. Zürcher, Rationale Normalformen von pseudo-linearen Abbildungen, Master's thesis, Mathematik, ETH Zürich (1994);

S. Gerhold, Uncoupling systems of linear Ore operator equations, Master's thesis, RISC,

J. Kepler University, Linz, 2002;

C. Schneider, A. De Freitas and J. Blümlein, PoS LL 2014 (2014) 017 [arXiv:1407.2537 [cs.SC]].

[56] C. Schneider, Sém. Lothar. Combin. 56 (2007) 1, article B56b.

[57] C. Schneider, Simplifying Multiple Sums in Difference Fields, in: Computer Algebra in Quantum Field Theory: Integration, Summation and Special Functions Texts and Monographs in Symbolic Computation eds. C. Schneider and J. Blümlein (Springer, Wien, 2013) 325 [arXiv:1304.4134 [cs.SC]].

[58] G. Almkvist and D. Zeilberger, J. Symb. Comp. 10 (1990) 571.

M. Apagodu and D. Zeilberger, Adv. Appl. Math. (Special Regev Issue), 37 (2006) 139.

[59] M. Karr, J. ACM 28 (1981) 305.

[60] C. Schneider, Symbolic Summation in Difference Fields Ph.D. Thesis RISC, Johannes Kepler University, Linz technical report 01-17 (2001).

[61] C. Schneider, An. Univ. Timisoara Ser. Mat.-Inform. 42 (2004) 163;

J. Differ. Equations Appl. 11 (2005) 799;

Appl. Algebra Engrg. Comm. Comput. 16(2005) 1.

[62] C. Schneider, J. Algebra Appl. 6 (2007) 415.

[63] C. Schneider, Motives, Quantum Field Theory, and Pseudodifferential Operators (Clay Mathematics Proceedings Vol. 12 ed. A. Carey, D. Ellwood, S. Paycha and S. Rosenberg,(Amer. Math. Soc) (2010), 285 [arXiv:0904.2323].

[64] C. Schneider, Ann. Comb. 14 (2010) 533 [arXiv:0808.2596].

[65] C. Schneider, in: Computer Algebra and Polynomials, Applications of Algebra and Number Theory, J. Gutierrez, J. Schicho, M. Weimann (ed.), Lecture Notes in Computer Science (LNCS) 8942 (2015), 157[arXiv:13077887 [cs.SC]].

[66] C. Schneider, J. Symbolic Comput. 43 (2008) 611,[arXiv:0808.2543v1];

J. Symb. Comput. 72 (2016) 82, [arXiv:1408.2776 [cs.SC]].

[67] C. Schneider, Ann. Comb. 9(1) (2005) 75;

S.A. Abramov and M. Petkovšek, J. Symbolic Comput., 45(6) (2010) 684;

C. Schneider, Appl. Algebra Engrg. Comm. Comput., 21(1) (2010) 1;

C. Schneider, In: Symbolic and Numeric Algorithms for Scientific Computing (SYNASC), 2014, 15th International Symposium, F. Winkler, V. Negru, T. Ida, T. Jebelean, D. Petcu, S. Watt, D. Zaharie (ed.), (2015) pp. 26; IEEE Computer Society, arXiv:1412.2782v1 [cs.SC]. 
[68] J. Ablinger, J. Blümlein, S. Klein and C. Schneider, Nucl. Phys. Proc. Suppl. 205-206 (2010) 110 [arXiv:1006.4797 [math-ph]];

J. Blümlein, A. Hasselhuhn and C. Schneider, PoS (RADCOR 2011) 032 [arXiv:1202.4303

[math-ph]];

C. Schneider, Computer Algebra Rundbrief 53 (2013), 8;

C. Schneider, J. Phys. Conf. Ser. 523 (2014) 012037 [arXiv:1310.0160 [cs.SC]].

[69] C. Schneider, Advances in Applied Math. 34(4) (2005) 740;

J. Ablinger, J. Blümlein, M. Round and C. Schneider, PoS(LL2012)050, (2012) 14 p.

[arXiv:1210.1685 [cs.SC]];

M. Round et al., in preparation.

[70] J. Ablinger, Ph.D. Thesis, J. Kepler University Linz, 2012, arXiv:1305.0687 [math-ph];

[71] J. Ablinger, PoS LL 2014 (2014) 019; J. Ablinger, A Computer Algebra Toolbox for Harmonic Sums Related to Particle Physics, Diploma Thesis, J. Kepler University Linz, 2009, arXiv:1011.1176 [math-ph].

[72] S. Alekhin, J. Blümlein and S. Moch, Phys. Rev. D 89 (2014) 5, 054028 [arXiv:1310.3059 [hep-ph]].

[73] S. Catani, M. Ciafaloni and F. Hautmann, Nucl. Phys. B 366 (1991) 135.

[74] H. Kawamura, N.A. Lo Presti, S. Moch and A. Vogt, Nucl. Phys. B 864 (2012) 399 [arXiv:1205.5727 [hep-ph]].

[75] J. Blümlein and A. Vogt, Phys. Rev. D 58 (1998) 014020 [hep-ph/9712546].

[76] J. Ablinger, J. Blümlein, S. Klein, C. Schneider and F. Wißbrock, arXiv:1106.5937 [hep-ph]; J. Ablinger, J. Blümlein, A. Hasselhuhn, S. Klein, C. Schneider and F. Wißbrock, PoS (RADCOR2011) 031 [arXiv:1202.2700 [hep-ph]];

[77] R. Harlander, T. Seidensticker and M. Steinhauser, Phys. Lett. B 426 (1998) 125 [hep-ph/9712228]; T. Seidensticker, hep-ph/9905298.

[78] J. Ablinger et al., DESY 14-019. 